\title{
Justiça social e lei dos cuidados inversos
}

Paula Broeiro*

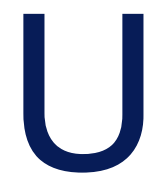

m estudo recente, realizado nos Estados Unidos e publicado na JAMA, revelou que rendimentos mais elevados parecem estar associados a maior longevidade; contudo, pouco se sabe sobre o mecanismo exato dessa associação. ${ }^{1}$ Apesar das limitações identificadas no estudo verificou-se que, relativamente à esperança de vida: esta aumentou de forma contínua com o rendimento; a sua desigualdade em populações de rendimentos diferentes aumentou nos últimos anos; verifica-se grande variação entre locais. ${ }^{1}$ Esta variação da esperança de vida entre locais foi utilizada como uma lente para avaliar as teorias que revelam diferenças socioeconómicas na longevidade, nomeadamente para compreender as características dos indivíduos que vivem mais. Nas áreas de baixo rendimento, a esperança de vida esteve altamente correlacionada com comportamentos (tabagismo, obesidade e exercício) e não correlacionada com medidas de quantidade e qualidade dos cuidados médicos. ${ }^{1}$ Um dado interessante foi que os indivíduos de baixo rendimento tendem a viver mais tempo em cidades com populações altamente qualificadas e elevados rendimentos. Desconhece-se o mecanismo porque ocorre, questionando-se se comportamentos saudáveis (e.g., publicidade) influenciem uma experiência mimética. ${ }^{1}$ A forte associação entre a variação geográfica da esperança de vida e comportamentos saudáveis sugere que as intervenções políticas devem concentrar-se na mudança de comportamentos e na redução das disparidades, o que pode exigir políticas locais. ${ }^{1}$

Estes resultados vêm confirmar o que os estudos epidemiológicos do cancro já haviam demonstrado para a doença oncológica, o reflexo dos determinantes sociais de saúde na sobrevivência. Em Inglaterra e no País de Gales, durante a década de oitenta, a sobrevida aos cinco anos para os indivíduos com cancro que viviam em áreas mais carenciadas foi significativamente menor do que para os que residiam em áreas mais abastadas. ${ }^{2}$ Num estudo de Carnon e colaboradores, mulheres com cancro da mama,

*Directora da Revista Portuguesa de Medicina Geral e Familiar residentes em áreas de baixo nível socioeconómico, tiveram sobrevivência significativamente menor do que as residentes em áreas ricas. ${ }^{3} \mathrm{~A}$ associação não foi significativa entre privação socioeconómica e fatores de prognóstico tumoral (comprometimento axilar, grau de diferenciação histológica, concentração de recetor de estrogénio, estadio do tumor ou biologia). ${ }^{3}$ São, pois, necessárias outras explicações para as diferenças de sobrevivência, como: diversidade de tratamento, resposta do hospedeiro, tempo de vida com qualidade ${ }^{4}$ ou se a elevada incidência e sobrevida de cancro em regiões desenvolvidas não se deve ao sobrediagnóstico (e.g., próstata). ${ }^{4}$

As diferenças em saúde relacionadas com o gradiente social também se encontraram num estudo escocês sobre multimorbilidade cujos autores concluíram que esta não era simplesmente uma consequência cronológica do envelhecimento - estava associada a determinantes sociais de saúde. ${ }^{5}$ Metade das pessoas com multimorbilidade tinha idade inferior a 65 anos e, em áreas carenciadas, esta ocorria cerca de 10 a 15 anos antes, existindo, nesta população, uma associação frequente entre problemas físicos e mentais, o que significa maior complexidade clínica requerendo cuidado holístico. ${ }^{5}$

As evidências levam-nos a questionar as políticas e a alocação de recursos em saúde quase quarenta anos após a Conferência de Alma-Alta (1978). Um estudo populacional recentemente publicado na Lancet veio revelar o efeito, a longo prazo, sobre a mortalidade, da remuneração por desempenho em cuidados de saúde primários (CSP). O objetivo do estudo foi verificar se o programa de pagamento por desempenho estava associado a redução na mortalidade da população. ${ }^{6}$ Comparando as alterações nas taxas de mortalidade, para as doenças incluídas no programa não se verificou diminuição significativa. ${ }^{6}$ No Reino Unido, o pagamento por desempenho parece não ser um método eficaz para a melhoria da mortalidade da população, devendo o custo-efetividade do programa ser comparado com outras intervenções. ${ }^{6}$

Segundo o Relatório de Marmot, para se conseguir melhorar a saúde e bem-estar de todos e reduzir as de- 


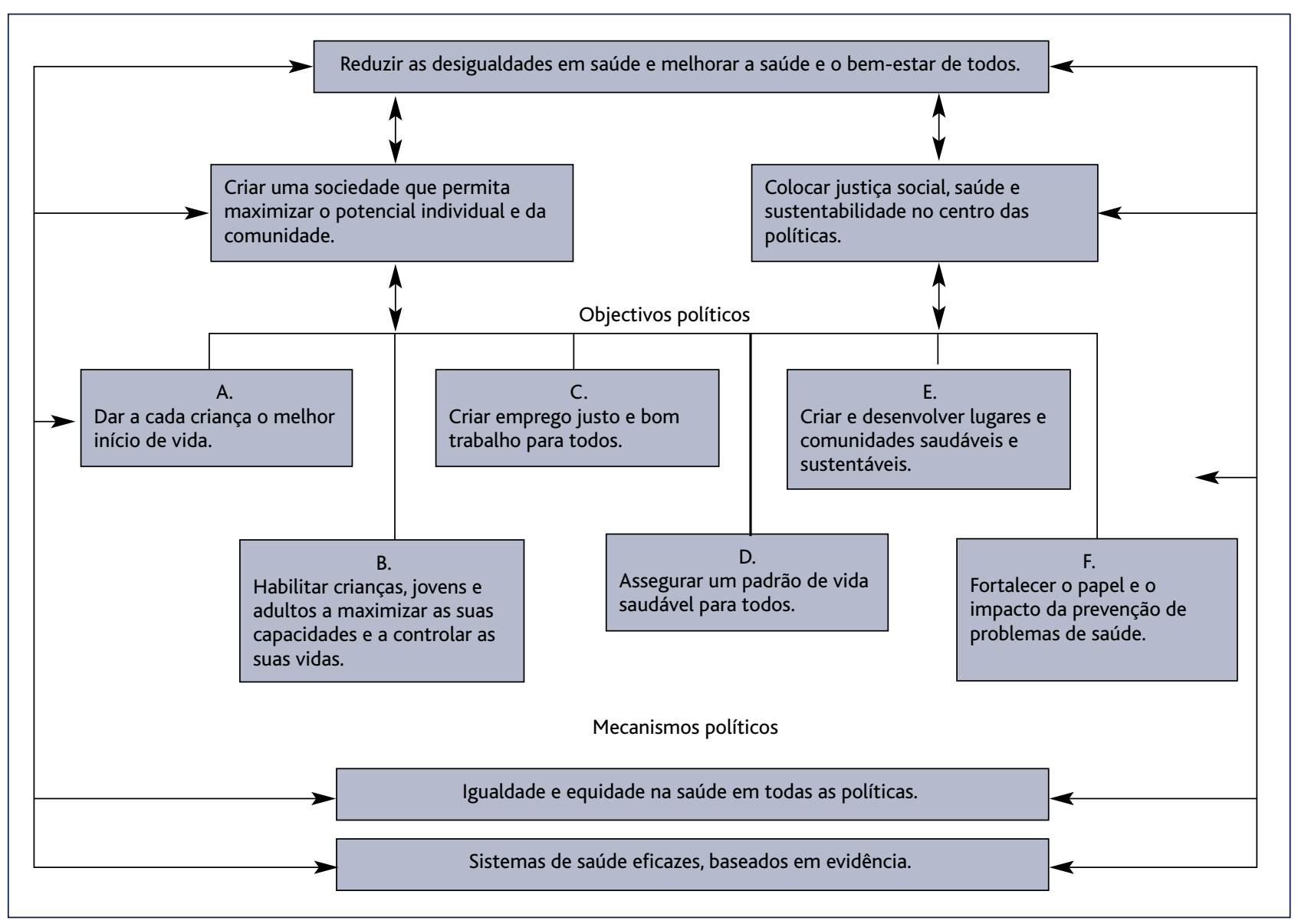

Figura 1. Estrutura concetual de redução das desigualdades em saúde. ${ }^{* 7}$

*Publicado com autorização de Sir Michael Marmot.

sigualdades em saúde (Figura 1), temos de conseguir dois objetivos políticos: 1) criar uma sociedade que permita maximizar o potencial individual e o potencial da comunidade e 2) fazer existir no centro de todas as políticas a garantia de justiça social e de saúde sustentável. ${ }^{7}$

As estratégias políticas sugeridas por Marmot vêm de encontro aos valores fundamentais da saúde pública europeia que incluem os três grandes princípios éticos: liberdade, igualdade e fraternidade. A solidariedade é o valor moral que melhor define o conceito europeu de saúde pública e é expressa como o bem comum e a responsabilidade coletiva. ${ }^{8}$ Considerando que a vulnerabilidade está associada a problemas de saúde, a ética da saúde pública dá especial ênfase a áreas carenciadas e à redução das desigualdades sociais. ${ }^{8}$ Estes modelos de bem-fazer são os que inspiraram o desenvolvimento de sistemas de saúde universais (e.g., português e inglês). ${ }^{8}$ Contudo, devido à crise económica, ao envelhecimento e à tendência para o individualismo excessivo, os princípios éticos europeus vêm sendo contrariados pelas atuais lógicas de gestão inspiradas em princípios éticos utilitaristas importados da escola americana. ${ }^{8}$

Relativamente ao Serviço Nacional de Saúde (SNS), a OCDE reconheceu que Portugal possui um SNS com arquitetura robusta que, ao contrário de muitos países da OCDE, cobre todo o país. ${ }^{9} \mathrm{~A}$ aparente bem-sucedida reforma dos CSP tornou assimétricos os cuidados em que apenas metade da população tem acesso a cuidados de qualidade superior. ${ }^{9}$ Do ponto de vista da equidade é necessária uma reflexão estratégica em torno do equilíbrio entre as unidades tradicionais de cuidados de saúde primários (UCSP) e as Unidades de Saúde da Família (USF), 
de forma a garantir cuidados de alta qualidade a toda a população. ${ }^{9}$ Com a reforma nacional dos CSP, contemporânea duma depressão no número de médicos de família e de uma grave crise económica, retornou-se à lei dos cuidados inversos de Tudor-Hart que significa que $a$ disponibilidade de bons cuidados médicos tende a variar inversamente com a necessidade da população abrangi$d a .^{10} \mathrm{~A}$ lei dos cuidados inversos não é uma lei natural, é muito sensível às forças do mercado ${ }^{10-11}$ e é o resultado de políticas que restringem o acesso a cuidados básicos efetivos. ${ }^{11}$ Em áreas com maior carga de doença, os médicos de família têm mais trabalho, listas maiores, menos suporte hospitalar do que nas áreas mais saudáveis. ${ }^{10}$ Mesmo no Reino Unido, onde existe uma cobertura universal de médicos de família, verifica-se desigualdade na sua distribuição, uma vez que a colocação é baseada na dimensão da população e não nas suas necessidades. ${ }^{11-12}$ Mais, a lei dos cuidados inversos afeta diretamente a consulta, uma vez que os doentes e os problemas são mais complexos e os médicos menos preparados. ${ }^{11}$ Embora os principais determinantes sociais da saúde operem fora dos cuidados de saúde, os cuidados de saúde podem mitigar os efeitos das más condições de saúde, reduzindo a gravidade e/ou retardando a progressão da doença. ${ }^{12}$

Em Portugal, nos CSP, a discussão tem-se centrado na carência de médicos de família, mas torna-se necessário um olhar atento sobre a contribuição de outros prestadores de saúde (e.g., enfermeiros). A complementaridade de funções e competências entre médicos e enfermeiros, associada a um rácio equilibrado (enfermeiro/médico) poderia trazer ganhos potencialmente relevantes na capacidade e qualidade da prestação de cuidados, ${ }^{9}$ ao invés de gastos com a subcontratação de médicos indiferenciados através de empresas de prestação de serviços.

Retornando a Marmot, ${ }^{7}$ as políticas de saúde devem garantir justiça social, visar a melhoria da saúde das populações, capacitando a comunidade, investindo na melhoria das condições de risco (desigualdades) e não apenas no controlo de fatores de risco (cuidados de saúde).

\section{REFERÊNCIAS BIBLIOGRÁFICAS}

1. Chetty R, Stepner M, Abraham S, Lin S, Scuderi B, Turner N, et al. The association between income and life expectancy in the United States, 2001-2014. JAMA. 2016;315(16):1750-66.

2. Coleman MP, Babb P, Sloggett A, Quinn M, De Stavola B. Socioeconomic inequalities in cancer survival in England and Wales. Cancer. 2001; 91(1 Suppl):208-16.

3. Carnon AG, Ssemwogerere A, Lamont DW, Hole DJ, Mallon EA, George WD, et al. Relation between socioeconomic deprivation and pathological prognostic factors in women with breast cancer. BMJ. 1994;309 (6961):1054-7.

4. Ferlay J, Soerjomataram I, Dikshit R, Eser S, Mathers C, Rebelo M, et al. Cancer incidence and mortality worldwide: sources, methods and major patterns in GLOBOCAN 2012. Int J Cancer. 2015;136(5):E359-86.

5. Barnett K, Mercer SW, Norbury M, Watt G, Wyke S, Guthrie B. Epidemiology of multimorbidity and implications for health care, research, and medical education: a cross-sectional study. Lancet. 2012;380 (9836):37-43.

6. Ryan AM, Krinsky S, Kontopantelis E, Doran T. Long-term evidence for the eff ect of pay-for-performance in primary care on mortality in the UK: a population study. Lancet. 2016 May 17. doi: 10.1016/S01406736(16)00276-2

7. Caan W. Fair society, healthy lives: timing is everything. BMJ. 2010; 340:c1191.

8. Camps V, Hernández-Aguado I, Puyol A, Segura A. An ethics training specific for European public health. Public Health Rev. 2015;36:6.

9. OECD. OECD reviews of health care quality: Portugal 2015 [Internet]. Paris: OECD; 2015. ISBN 9789264225985. Available from: http:// www.oecd-ilibrary.org/content/book/9789264202054-en

10. Hart JT. The inverse care law. Lancet. 1971;297(7696):405-12.

11. Mercer SW, Guthrie B, Furler J, Watt GC, Hart JT. Multimorbidity and the inverse care law in primary care. BMJ. 2012;344:e4152.

12. McLean G, Guthrie B, Mercer SW, Watt GC. General practice funding underpins the persistence of the inverse care law: cross-sectional study in Scotland. Br J Gen Pract. 2015;65(641):e799-805.

\section{ENDEREÇO PARA CORRESPONDÊNCIA}

director@rpmgf.pt 